\title{
On the of classification of BL Her subtype of the Type II Cepheids
}

\author{
Monika I. Jurkovic ${ }^{\mathrm{a}}$ \\ Astronomical Observatory of Belgrade, Volgina 7, 11060 Belgrade, Serbia
}

\begin{abstract}
The initial reason for studying Type II Cepheids (CWB) was for period changes, binarity or any other signs of change other than the pulsation. The GCVS contained 71 objects of this kind in 2012 when the wok started, and that list is now extended to $100 \mathrm{CWB}$ and $26 \mathrm{CWB}$ : objects. The photometric data for the 71 objects was collected from ASAS, AAVSO, CATALINA, LINEAR, SuperWASP, NSVS surveys. Surprisingly, there is a discrepancy in the classification of the stars in this list. A large percentage of these objects might not be not Type II Cepheids, some of them are classical Cepheids, RR Lyrae, some eclipsing binaries, there is even a dwarf novae.
\end{abstract}

\section{Introduction}

Type II Cepheids (CWB) are considered to be important distance indicators together with the classical Cepheids. On the PL diagram they appear to have magnitudes lower than Classical Cepheids of about 0.5 mag. The nature of their pulsation is usually explained in the same way as in RR Lyrae stars: radially pulsating metal deficient objects crossing the instability strip (e. g. [6]). The best studied examples are the LMC and SMC, but they are also known in globular clusters.

\section{Data sample and analysis}

The studied sample contains 71 objects, which were taken from the General Catalogue of Variable Stars (GCVS) in 2012. They were all listed as BL Her subtype. The present list at GCVS contains 126 objects, but this research has not yet been extended to all of them . The data for each object was collected from the American Association of Variable Star Observers ([3]), All Sky Automated Survey ([5]), the CATALINA Sky Survey ([2]), INTEGRAL-OMC Archive, LINEAR (http://skydot.lanl.gov/), SuperWASP ([1]), the North Sky Variability Survey (http://skydot.lanl.gov/nsvs/nsvs.php). Additional information from the literature was also gathered for each object (e. g. $[\mathrm{Fe} / \mathrm{H}], T_{\text {eff } \ldots) \text {. }}$

The photometric data were all transformed to magnitudes, and then analysed using Fourier series in the form: $A_{0}+\sum_{i=1}^{\infty} \cos 2 \pi\left[i f_{i}\left(t-t_{0}\right)+\varphi_{i}\right]$, where $A_{i}$ is the amplitude, $f_{i}$ is the frequency, $\varphi_{i}$ is the phase. The basic period (P) was also determined from the data points, and compared to the literature data. Period04 ([4]) was used to perform this analysis. A Petersen diagram (see Fig 1) was created using the following parameters: $R_{21}=A_{2} / A_{1}$ vs $\log P$. For the light curve shape classification the GCVS Variablilty Type (www.sai.msu.su/gcvs/gcvs/iii/vartype.txt) was followed.

\footnotetext{
a e-mail: mojur@aob.rs
}

This is an Open Access article distributed under the terms of the Creative Commons Attribution License 4.0, which permits unrestricted use, distribution, and reproduction in any medium, provided the original work is properly cited. 
Fig. 1. Possible RR Lyrae $\left(\log P, R_{21},+\right)$ : FY Aqr (0.00984, 0415), FY Vir $(0.03419,0.4472)$, PP Tel $(0.03769,0.5962)$, DF Hyi $(0.05023,0.5378)$, BF Ser $(0.06649,0.4889)$, BI Tel $(0.06685,0.5071)$, CE Her (0.08257, 0.4595), VX Cap $(0.12305,0.4273)$, XX Vir (0.12976, 0.3867), Type II Cepheids $\left(\log P, R_{21}, \mathrm{x}\right)$ : BX Del $(0.038142,0.3926)$, GP Aur $(0.22268,0.1771)$, BV Cha (0.09273, 0.3373), BL Her $(0.11642,0.3570)$, MQ Aql $(0.170464,06253)$, SW Tau $(0.19962,0.3181)$, V477 Oph (0.30442, 0.3891)

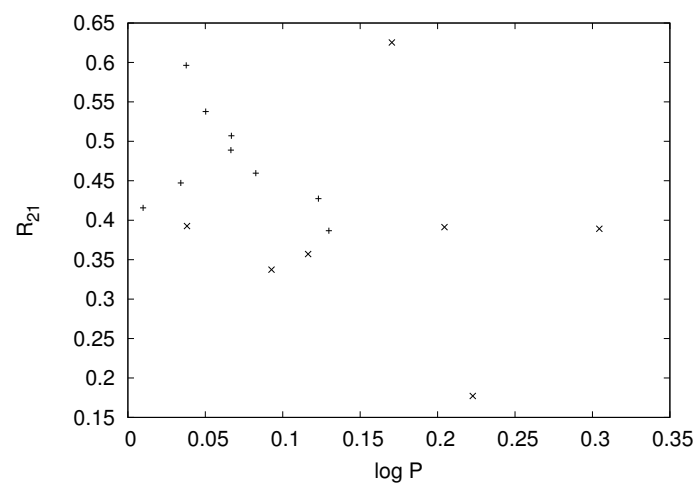

\section{Results}

\subsection{No data or insufficient data}

For the following stars no data was found or the number of data points was not enough to do the Fourier analysis: V403 Cyg, V742 Cyg, WY CMa, V1153 Sgr, V553 Sco, AT Tel, HQ CrA, YZ CMa, V839 Sgr, V714 Cyg, VX CMa, BE Pup, V351 Cep, BE CrA, LN Pav, QY Cyg, KO Lyr.

\subsection{Misclassified objects}

The following list contains the objects which are not CWBs: V2022 Sgr, V4110 Sgr, FM Del, NY Her, UW For, KT Com.

\section{Conclusion}

The presented results are not final, and this research is still ongoing. Fig 1 shows a possible solution of the discrepancies seen during the analysis of the stars - some could be RR Lyrae type variables. 32 objects were not presented here, but many of them are possible classical Cepheids. From the examined stars two things became clear, despite them being known bright variables: (i) the clarification and unification of classification criteria would be useful; (ii) additional multi-color photometric and detailed spectroscopic measurements are needed.

I would like to thank for the grant from the conference organizers. The work was supported by the project number 176004 of the Serbian Ministry of Education, Technical Development and Science. The research was supported by the DOMUS program of the Hungarian Academy of Sciences. This research has made use of the SIMBAD and VizieR catalogue access tool, CDS, Strasbourg, France. Based on data from the OMC Archive at CAB (INTACSIC), pre-processed by ISDC.

\section{References}

1. Butters, O. W., West, R. G., Anderson, D. R., et al., A\& A 520, (2010) LL10

2. Drake, A. J., Djorgovski, S. G., Mahabal, A., et al., ApJ 696, (2009) 870-884

3. Henden, A. A., Observations from the AAVSO International Database, http://www.aavso.org

4. Lenz P., Breger M., CoAst 146, (2005) 53

5. Pojmanski, G., Acta Astronomica 52, (2002) 397-427

6. Wallerstein, G., PASP 114, (2002) 689-699 